\title{
Interest of colchicine in the treatment of acute myocardial infarct responsible for heart failure in a mouse model
}

\author{
Mariama Akodad ${ }^{\mathrm{a}, \mathrm{b}}$, Jérémy Fauconnier ${ }^{\mathrm{b}}$, Pierre Sicard ${ }^{\mathrm{b}}$, Fabien Huet ${ }^{\mathrm{a}, \mathrm{b}}$, Florence Blandel ${ }^{\mathrm{b}}$, Annick Bourret ${ }^{\mathrm{b}}$, \\ Pascal de Santa Barbara ${ }^{\mathrm{b}}$, Sylvain Aguilhon ${ }^{\text {a }}$, Marion LeGall ${ }^{\mathrm{b}}$, Gérald Hugon ${ }^{\mathrm{b}}$, \\ Alain Lacampagne ${ }^{\mathrm{b}}$, François Roubille ${ }^{\mathrm{a}, \mathrm{b}, *}$ \\ a Cardiology Department, University Hospital of Montpellier, Montpellier, France \\ b PhyMedExp, University of Montpellier, INSERM U1046, CNRS UMR 9214, 34295 Montpellier cedex 5, France
}

\begin{abstract}
A B S T R A C T
Background: Inflammation is deeply involved in the pathophysiology of ischemia-reperfusion (I/R) lesions and ventricular remodeling due to an acute myocardial infarction (AMI). Colchicine as a pleiotropic antiinflammatory molecule may exert cardioprotective effects under acute ischemia. Here, we aimed to evaluate the impact of colchicine on reperfusion injury in a mouse model.

Method: Myocardial ischemia/reperfusion (I/R) injury was induced in C57BL/6 male mice, after 45 min ligation of the left coronary artery followed by reperfusion. $400 \mu \mathrm{g} / \mathrm{kg}$ of colchicine or the vehicle was administrated intraperitoneally (i.p.) 25 min before the reperfusion (blinded administration). Mice were sacrificed at $24 \mathrm{~h}$ after the acute myocardial ischemia (AMI) and the infarct size was determined. Circulating level of troponin and cytokines profile were assessed $4 \mathrm{~h}$ after the AMI. An echocardiography was performed in a follow-up group mice, $48 \mathrm{~h}$ and 8 weeks after the AMI.

Results: The infarct size was reduced in colchicine treated mice ( $39.8 \pm 3.5 \%$ versus $52.9 \pm 3.2 \%, \mathrm{p}<0.05$ ). Troponin was significantly lower in the colchicine treated mice $(7015.7 \pm 1423.7 \mathrm{pg} / \mathrm{mL}, \mathrm{n}=5$ vs 30,723.7 \pm $7959.9 \mathrm{pg} / \mathrm{mL}$ in the placebo group, $\mathrm{n}=6 ; \mathrm{p}<0.0001)$.

Fibrosis was decreased in the Colchicine group $(24.51 \pm 3.13 \%$ vs $11.38 \pm 2.46 \%, \mathrm{p}=0.03)$. In the follow-up group mice $(n=8)$, there were no differences between mice treated with placebo $(n=9)$ and mice treated with colchicine $(n=9)$ regarding to cardiac remodeling parameters but outflow approximated by the ITV was higher in the colchicine group.

Conclusion: In conclusion, colchicine allowed a significant reduction of infarct size in mice, improves hemodynamic parameters and decrease cardiac fibrosis.
\end{abstract}

\section{Introduction}

Ischemic heart failure (HF) is a progressive disorder characterized by poor quality of life, a poor prognosis ( 5 -year survival $<50 \%$, worse than most of common types of cancers), and a tremendous burden on health care costs [1]. In Europe and the United States $\sim 1-2 \%$ of the entire health care budget is spent on HF. The prevalence of HF is expected to rise due to the aging population and better treatment of cardiovascular disease

Abbreviations: AAR, area at risk; AMI, acute myocardial infarction; CAD, coronary artery disease; HF, Heart failure; IA, infarcted area; IR, ischemia-reperfusion; LVEDV, left ventricular end-diastolic volume; LVEF, left ventricular ejection fraction; MRI, magnetic resonance imaging; RAS, renin-angiotensin system; SNS, sympathetic nervous system; STEMI, ST elevation myocardial infarction; TTC, Triphenyl-tetrazolium chloride.

* Corresponding author at: Cardiology Department, University Hospital of Montpellier, Montpellier, France.

E-mail address: francois.roubille@gmail.com (F. Roubille). that precedes HF [2]. Therefore, it is mandatory to explore innovative approaches. Over the last decade, among the pathophysiological mechanisms associated with ischemic HF progression, inflammatory processes appear appealling to define new therapeutic strategies [3-5]. Indeed, after an acute myocardial infarction, inflammation could be involved at least at two levels, by worsening the infarct size at the very onset of the reperfusion, or at later stages by worsening the cardiac remodeling [4, 5].

Cardiac remodeling is a crucial determinant of the clinical outcome of HF and is linked to disease progression and poor prognosis. The remodeling process is characterized by activation of "compensatory" systems, including the renin-angiotensin system (RAS) and the sympathetic nervous system (SNS). Although initially aimed at maintaining adequate circulation, over time the sustained activation of compensatory neurohormonal systems actually contributes to the adverse remodeling process leading to HF. In parallel, cardiac remodeling is 
accompanied by a progressive inflammatory response characterized by inflammatory cells infiltration and pro-inflammatory cytokines, which favors fibrosis expansion [6]. As such, the severity of ischemic HF is positively correlated to the inflammatory profile [7]. Despite current treatment regimens for HF that effectively target known neurohormonal system activation, clinical outcomes remain poor. Other targets, such as cardiac fibrosis, are currently left untreated [8].

Colchicine is a mitotic spindle poison used for centuries for the treatment and prevention of gouty attacks and rheumatic complaints and is one of the oldest drugs still currently available [9]. It could exert pleiotropic anti-inflammatory effects. Especially, colchicine has direct antiinflammatory effects [10] by inhibiting key inflammatory signaling networks as the inflammasome, pro-inflammatory cytokines and expression of adhesion molecules, preventing both local chemoattraction of inflammatory cells such as neutrophils [11] and systemic inflammation including the decrease of release of IL-1 $\beta$ by the neutrophils [12-14]. At the cellular level, colchicine could also exert antiarrhythmic effects [15]. In in vivo models, colchicine has been demonstrated to inhibit apoptosis in rats [16] and to exert indirect antifibrotic effects, by inhibiting the release of profibrotic factors [17]. Although, in a dog model subjected to a 120 -min coronary artery occlusion followed by 6 -h reperfusion [18], IV injection of colchicine reduce post-ischemic myocardial neutrophil accumulation, no myocardial protection could be detected in terms of infarct size. More recently colchicine has been proposed to reduce infarct size in patients [19] suggesting that colchicine could be a potential therapeutic strategy for treatment of heart failure induced by acute myocardial infarct. However, the cardioprotective impact of colchicine remains under debate.

Thus, the objective of this study was to evaluate the impact of colchicine on infarct size and cardiac remodeling in a mice model of acute myocardial infarction. We demonstrated that colchicine reduced myocardial infarct size and left ventricular remodeling was accompanied by a profound anti-inflammatory effects.

\section{Method}

\subsection{Experimental mode}

Eight to ten weeks C57BL/6 male mice, were randomized into 2 groups: colchicine or placebo. Myocardial ischemia-reperfusion (IR) injuries were induced in all mice under general anesthesia with intramuscular injection of ketamine $(50 \mathrm{mg} / \mathrm{kg}$ ) and xylazine $(10 \mathrm{mg} / \mathrm{kg})$ and after orotracheal intubation with a $22 \mathrm{G}$ venous catheter for controlled ventilation (Minivent, Harvard Apparatus) with controlled stroke $(10 \mu \mathrm{L} / \mathrm{g})$ and frequency $(150 / \mathrm{min})$. After left thoracotomy and muscular dissection, ligation of the left coronary artery was performed with a 8-0 silk and a smooth catheter was applied on the artery to obtain an ischemia for $45 \mathrm{~min}$. The ischemia was visually confirmed by the change in myocardial color turning into white and was followed by reperfusion obtained by the catheter removal. $400 \mu \mathrm{g} / \mathrm{kg}, 1 \mathrm{mg} / \mathrm{kg}$ and $2 \mathrm{mg} / \mathrm{kg}$ of colchicine or placebo was administrated intraperitoneally (i.p.) 25 min before the reperfusion (blinded administration) The determination of the dose is described elsewhere [20]. Muscle and cutaneous plans were sutured with silk 6-0. Mice were extubated and placed at $32{ }^{\circ} \mathrm{C}$ (RT) for $1 \mathrm{~h}$. Sham-operated animals were subjected to the same surgical procedure, but the ligation remained untied. This study was approved by the local ethic committee for animal experimentation and registered by the national committee under the number CEEA-LR-12079.

Sham-operated mice underwent the same procedure without the LAD occlusion/reperfusion and treated with saline $(\mathrm{n}=5)$ or colchicine $(400 \mu \mathrm{g} / \mathrm{kg}$, i.p.; $\mathrm{n}=5)$.

\subsection{Infarct size}

Twenty-four hours after IR, intracardiac Evans blue injection was performed ( $500 \mu \mathrm{L}$ ) with a $30 \mathrm{G} 1 / 2$ needle. Euthanasia was induced by intracardiac injection of $10 \%$ potassium chlorate.

The heart was then removed and the left ventricle (LV) was cut in $1 \mathrm{~mm}$ thick transverse slices, stained with Triphenyl-tetrazolium chloride $1.5 \%$ (TTC) and incubated at $37^{\circ}$ for $2 \mathrm{~h}$. The slices where then transferred in $0.9 \%$ saline serum at $4{ }^{\circ} \mathrm{C}$ and double blind analyzed with a binocular microscope $(\times 10)$. The area at risk (AAR) and infarcted area (IA) were determined by computerized planimetry with Imagej® software. The viable perfused myocardium was colored in blue, the IA in white and the ischemic viable myocardium in red. 5 slices were double blind analyzed on both side. The AAR was compounded by the IA and the ischemic but viable myocardium. The AAR/total area and IA/AAR ratios were calculated. Results are expressed in average of percentage of AAR on total area and percentage of IA on AAR, on the 5 slices.

\subsection{Blood analysis}

Twenty-four hours after reperfusion, a subgroup of mice was dedicated for blood analysis after intracardiac puncture under Isoflurane anesthesia. The blood was centrifuged $10 \mathrm{~min}$ at $5000 \mathrm{rpm}$ and the serum obtained was stored at $-20^{\circ} \mathrm{C}$. The myocardium injury biomarker $\mathrm{T}$ troponin and the major cytokines implicated in inflammatory process (IL1 $\beta$, IL6, IL10, CCL2-MCP1) were measured by Multiplex (Milliplex® MAP Millipore, Billerica, MA) following the manufacture indications. Briefly, 2 plates of 96 wells were used, one for the $\mathrm{T}$ troponin and one for the other cytokines assay. $125 \mu \mathrm{L}$ of serum was necessary per well, without dilution for $\mathrm{T}$ troponin dosage and with a 1:2 ratio dilution for the cytokines. The magnetic balls were prepared and incubated with serums overnight. The assays were triplicated for each serum with 2 negative controls by plate. The data were revealed by Luminex Multiplex assay.

\subsection{Transthoracic echocardiography}

A group of mice, after the same ischemia-reperfusion protocol was followed during 10 weeks.

An echocardiographic follow-up was performed 48 h, 14 days and 8 weeks after the infarction, under Isoflurane anesthesia with a maintained $36{ }^{\circ} \mathrm{C}$ body temperature and 450-500/min heart rate. The transthoracic echocardiography (Vevo2100; VisualSonics) were double blind realized, with a $40 \mathrm{MHz}$ probe, TM and 2D modes were used with long and short axis parasternal views. Left ventricular ejection fraction (LVEF), left ventricular end-systolic and end-diastolic diameter, E/A profile reflecting diastolic function and aortic ITV reflecting the cardiac output were assessed.

\subsection{Fibrosis study}

All the mice dedicated to the long-term follow-up were sacrificed 10 weeks after ischemia-reperfusion for fibrosis study. Briefly, the heart was removed and the left ventricle was separated. The left ventricle was embedded into paraffin and $8 \mu \mathrm{m}$ thick slices were obtained. The fibrotic area, in blue, was determined by Masson Trichrome (HT15 kit, Sigma Aldrich, France) and quantified using ImageJ. The percentage of total fibrosis area was calculated as the sum of blue-stained areas divided by total ventricular area.

\subsection{Clinical sub study}

A clinical trial [21] was conducted in parallel by our team and aimed to assess the impact of colchicine in post-myocardial infarction on inflammation, particularly on the peak of CRP. A sub-study was performed to investigate the impact of colchicine treatment on ventricular remodeling and to identify its predictive imaging parameters. A transthoracic echocardiography was performed in all patients included in this study. Treatment with colchicine was administered on the first day of the STEMI, for a period of 1 month at $1 \mathrm{mg}$ dose per day. The left ventricular remodeling was defined as the increase in left ventricular end-diastolic volume (LVEDV) $>20 \%$ at 1 month.

\subsection{Statistical analysis}

Statistical analyses were realized with GraphPad Prism (version 5, GraphPad software, La Jolla, CA). A Mann-Whitney test was used to compare the 2 groups of mice treated with colchicine or placebo for each analysis. The significance was fixed at $\mathrm{p}<0.05$. All data are expressed as percentage, mean and standard error of mean.

\section{Results}

\subsection{Toxicity and effect of colchicine on infarct size}

A preliminary phase aimed to identify the toxicity and the optimal dose of colchicine according to literature [18,20,22-24]. The ischemiareperfusion protocol was performed with a $2 \mathrm{mg} / \mathrm{kg}$ dose of colchicine in 2 animals, 3 received $1 \mathrm{mg} / \mathrm{kg}$ of colchicine and 9 mice $400 \mu \mathrm{g} / \mathrm{kg}$ of colchicine. The placebo (saline serum) was administrated in 10 mice. Higher dose $(\geq 1 \mathrm{mg} / \mathrm{kg}$ ) of colchicine were toxic for 3 mice $(60 \%)$ with early unexpected death $(<24 \mathrm{~h})$. The $400 \mu \mathrm{g} / \mathrm{kg}$ dose was then considered as optimal as none of them died prematurely. The two left mice treated with the higher dosage were excluded.

Twenty-six mice were sacrificed $24 \mathrm{~h}$ after ischemia for histological analysis, 13 in the placebo group and 13 in the colchicine group. Mean AAR/total area ratio were $52.6 \pm 1.1 \%$ in the colchicine group vs 50.6 $\pm 0.8 \%$ in the placebo group ( $\mathrm{p}=0.9$ ). Mean IA/AAR ratio were 39.8 $\pm 3.5 \%$ in the colchicine group vs $52.9 \pm 3.2 \%$ in the placebo group ( $\mathrm{p}$ $<0.05$ ), with a significant reduction of the infarct size in the colchicine group (Fig. 1, A, B, C). After $24 \mathrm{~h}$ after reperfusion, the T troponin level was significantly reduced in mice treated with colchicine (7015.7 \pm $1423.7 \mathrm{pg} / \mathrm{mL}, \mathrm{n}=5$ vs $30,723.7 \pm 7959.9 \mathrm{pg} / \mathrm{mL}$ in the placebo 
group, $\mathrm{n}=6 ; \mathrm{p}<0.0001$ ) (Fig. 1, D). Consequently, these results suggest that a low-dose of colchicine prior to reperfusion decreases myocardial injury and infarct size $24 \mathrm{~h}$ after an acute myocardial ischemia.

\subsection{Colchicine and inflammation}

To determine if the potential benefits of colchicine on myocardial injury was associated with an anti-inflammatory effect of the drug, the main inflammatory cytokines were measured $24 \mathrm{~h}$ after reperfusion (see Fig. 2). The IL6 level was $197.8 \pm 48.4 \mathrm{pg} / \mathrm{mL}$ in the colchicine group $(\mathrm{n}=5)$ vs $2303.0 \pm 1007.6 \mathrm{pg} / \mathrm{mL}$ in the placebo group $(\mathrm{n}=$ 6 ), $\mathrm{p}=0.0001$. The IL10 level was $20.2 \pm 18.2 \mathrm{pg} / \mathrm{mL}$ in the colchicine group $(n=5)$ vs $183 \pm 50.4 \mathrm{pg} / \mathrm{mL}$ in the placebo group $(\mathrm{n}=6), \mathrm{p}<$ 0.0001 . MCP-1 level was also significantly lower in the group treated with colchicine $(\mathrm{n}=5), 185.4 \pm 75.4 \mathrm{pg} / \mathrm{mL}$ vs $640 \pm 121 \mathrm{pg} / \mathrm{mL}$ in the placebo group $(n=6), p<0.0001$. The level of IL1 $\beta$ was not significant different between the 2 groups ( $p=0.3$, Fig. 2 ). Importantly, there was no significant differences in the Sham-groups. In conclusion, $24 \mathrm{~h}$ after ischemia-reperfusion, colchicine decreases the systemic proinflammatory cytokines.

\subsection{Colchicine and post-infarction cardiac remodeling}

To evaluate long-term effects of colchicine and the potential antifibrotic effect of the drug, a group of mice was followed during 10 weeks post-reperfusion. On 22 mice ( 11 in each group), 2 died before the 24th hour and 2 were excluded ( 1 in each group) for thoracotomy scar infection. The follow-up was realized for 9 mice in the colchicine group and 9 in the placebo group. All mice were sacrificed after 10 weeks to perform histological analyses. The initial body weight was similar between the two groups, $25.0 \pm 1.0 \mathrm{~g}$ in the colchicine group vs $26.3 \pm 2.0 \mathrm{~g}$ in the placebo group, $\mathrm{p}=0.5$ and there was no difference in terms of weight gain during the follow-up, $\mathrm{p}=0.6$.

Under echocardiography, the heart rate was comparable in both groups ( $531 \pm 10$ in the colchicine group vs $515 \pm 11$ in the placebo group, $\mathrm{p}=0.9$ ) and no significant difference was found for the $\operatorname{LVEF}(\mathrm{p}=0.2), \mathrm{E} / \mathrm{A}$ ratio $(\mathrm{p}=0.08)$, (Fig. $3 \mathrm{~A}$ and $\mathrm{B})$. The aortic ITV was significantly higher in mice treated with colchicine $(\mathrm{p}=$ 0.015 ) (Fig. $3 \mathrm{C}$ ) at $48 \mathrm{~h}$ and 14 days post reperfusion. After 8 weeks, aortic ITV remained significantly higher in the colchicine group, compared to the placebo group, $\mathrm{p}=0.014$ (Fig. $3 \mathrm{D}$ ). However, end-diastolic diameter ( $p=0.7$ ), end-systolic diameter ( $p$ $=0.2)$, LVEF ( $p=0.09)$, and E/A ratio $(p=0.9)$ (Fig. $3 C)$ were unchanged. Altogether this data indicates that colchicine improves cardiac hemodynamic parameters at long term with an improvement of cardiac output.

In the clinical sub-study, 44 patients were included, 23 received colchicine and 21 the optimal treatment alone. All the patients got complete and successful revascularization. Remodeling was observed in 12 patients, 4 patients of colchicine group $v s 8$ of the optimal treatment group alone. The percentage of necrosis was similar (independently of the remodeling). The percentage of variation in LVEDV was 6.8\% (95\% CI:[-3.1-16.6]; $\mathrm{p}=0.3$ ) for the colchicine group and $28.9 \%$ (95\% CI:[9.3-48.5]; $\mathrm{p}=0.4$ ) for the control group. The LVEDV was correlated
A
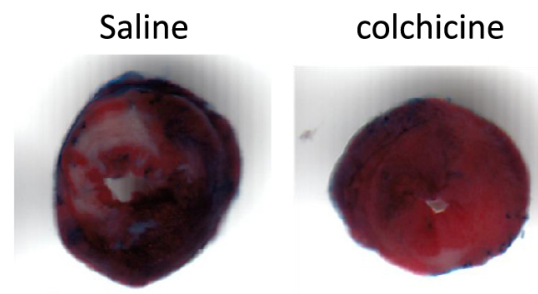

B

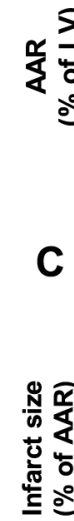

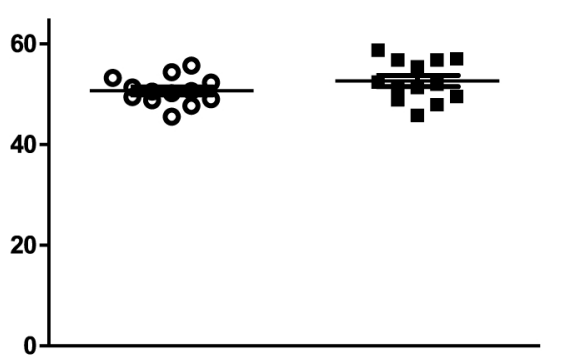

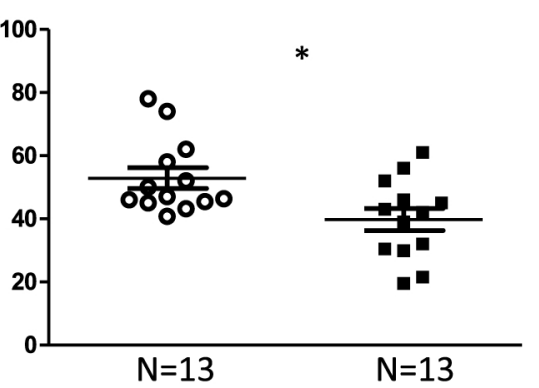

- Saline

- Colchicine

D

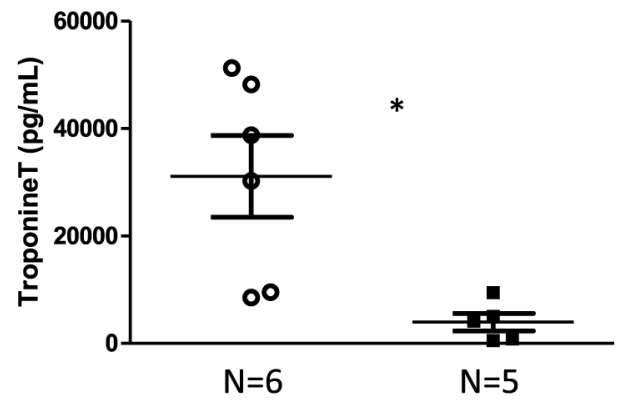

- Saline

- Colchicine
- Saline

- Colchicine

Fig. 1. Infarct size in mice treated with placebo (saline) or colchicine, $24 \mathrm{~h}$ after ischemia-reperfusion. A: Placebo and colchicine treated mice left ventricular slices after blue Evans injection and incubation in 2,3,5-triphenyltetrazolium (TTC), normal myocardium appears in deep blue, necrotic area in white and viable ischemic area in red. B: Area at risk (AAR)/total area of ventricular slice ratio in placebo and colchicine treated mice after blue Evans intracardiac injection and incubation in TTC. C: Infarct size/area at risk ratio in placebo and colchicine treated mice after blue Evans intracardiac injection and incubation in TTC. D: serum troponin T level (pg/mL) after intracardiac puncture in placebo and colchicine treated mice *p < 0.05 . (For interpretation of the references to color in this figure legend, the reader is referred to the web version of this article.) 

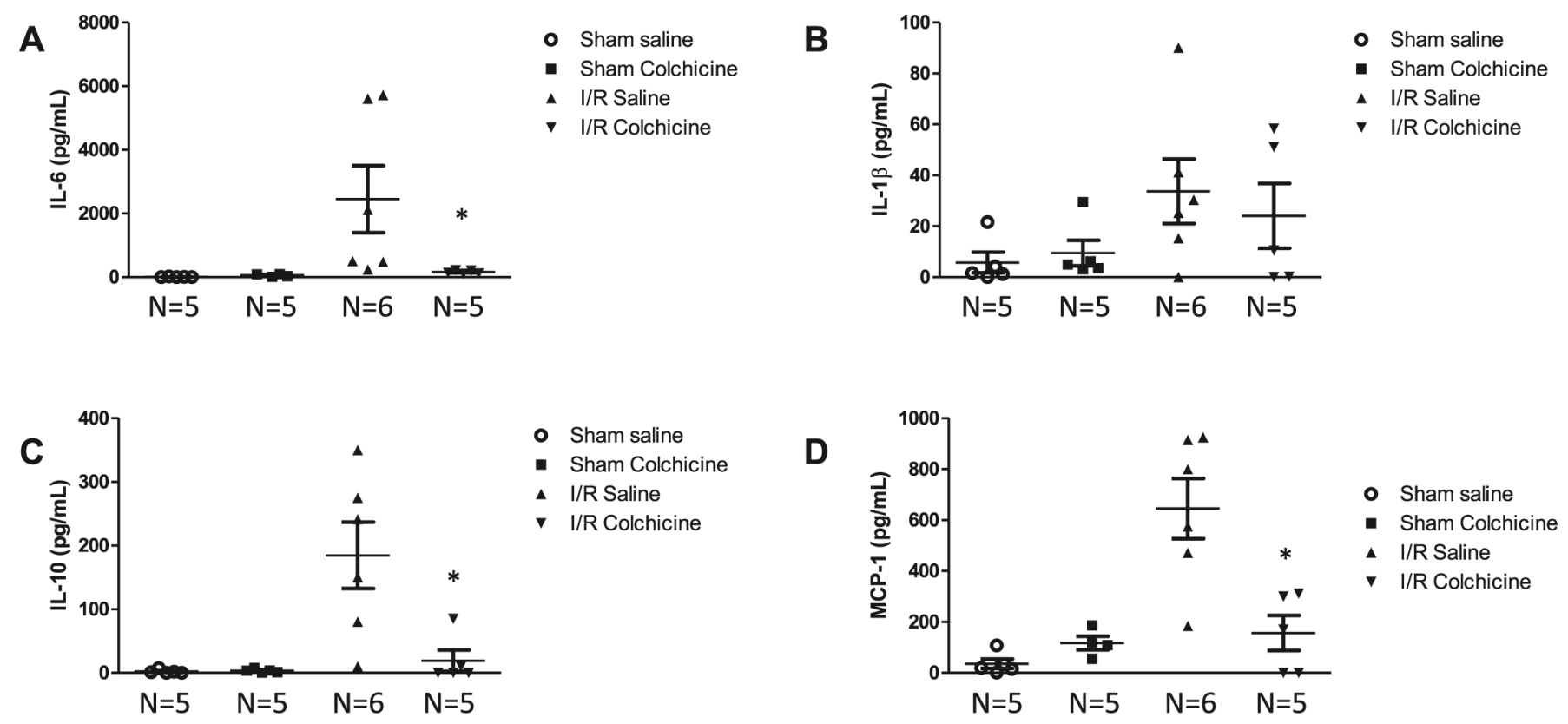

Fig. 2. Comparison of arterial inflammatory cytokines level, IL6 (A), IL1ß (B) IL10 (C) and MCP-1 (D) between the four groups, Sham-operated mice treated with saline (placebo) or colchicine and mice subjected to ischemia-reperfusion and treated with saline (placebo) or colchicine, respectively, after $24 \mathrm{~h}$ after Sham-surgery or ischemia-reperfusion and multiplex analysis. ${ }^{*} \mathrm{p}<0.05$.

to the remodeling, negatively at baseline (coefficient $-0.43 ; \mathrm{p}<0.05$ ), and positively at 1 month (coefficient $0.43 ; \mathrm{p}<0.05$ ). This data suggests that remodeling should be more present in the control group, corroborating the anti-remodeling impact of Colchicine in patients.

\section{A}

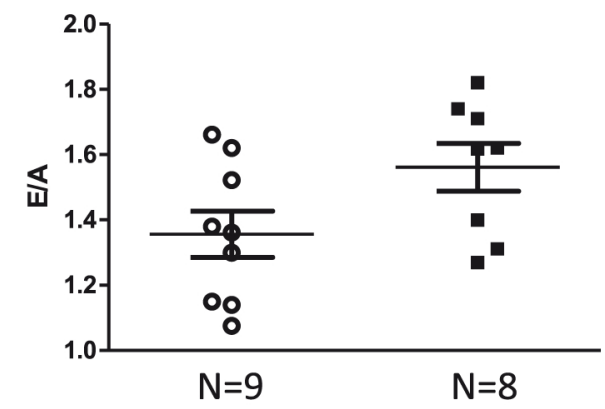

C

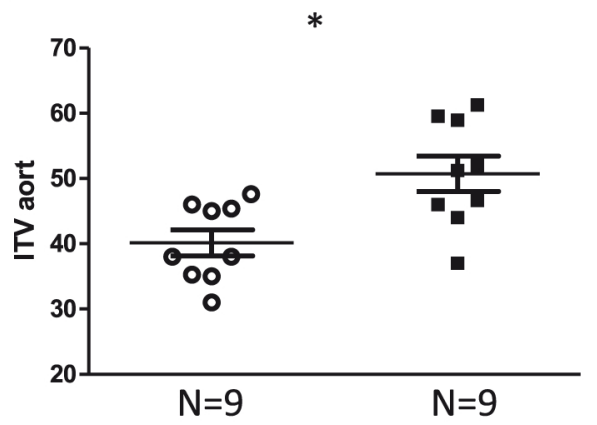

\section{8h post I/R}

- Saline

- Colchicine

- Saline

- Colchicine

\subsection{Colchicine and cardiac fibrosis}

Fibrosis was evaluated in 5 mice from the placebo group and 5 mice from the colchicine group.
B

8W post I/R

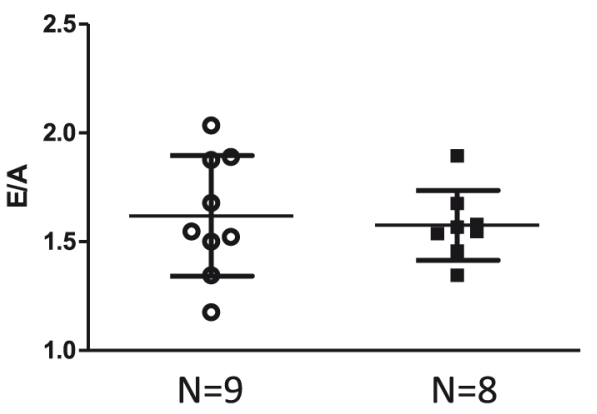

D

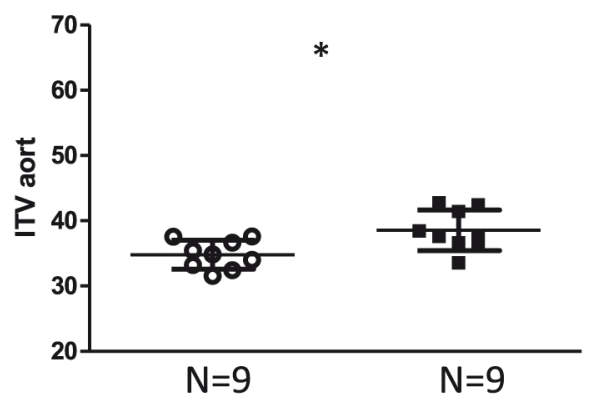

- Saline

- Colchicine

Fig. 3. Comparison of echocardiographic parameters between placebo and colchicine group mice. A: Comparison of E/A ratio between the two study groups $48 \mathrm{~h}$ after ischemiareperfusion. B: Comparison of aortic ITV between the two study groups $48 \mathrm{~h}$ after ischemia-reperfusion. C: Comparison of E/A ratio between the two study groups 8 weeks after ischemia-reperfusion. D: Comparison of aortic ITV between the two study groups 8 weeks after ischemia-reperfusion. *p $<0.05$. 
Area of myocardial fibrosis was significantly more important in the placebo group, $24.51 \pm 3.13 \%$ vs $11.38 \pm 2.46 \%$ in the colchicine group, $\mathrm{p}=0.03$, (Fig. 4).

\section{Discussion}

This study aimed to evaluate, the cardioprotective effect of colchicine in a mouse model of myocardial ischemia-reperfusion. Our data demonstrate that a treatment with colchicine is associated with 1) a decrease in infarct size, 2) a decrease in systemic inflammatory response and 3 ) an improvement in long term cardiac output and myocardial fibrosis.

A unique i.p. injection of colchicine $(0.4 \mathrm{mg} / \mathrm{kg})$, during ischemia, reduces infarct size (IA/AAR), and circulating T troponin level $24 \mathrm{~h}$ after ischemia-reperfusion, reflecting a reduction in myocardial injury. These data are in accordance with those obtained in a recent human study. Indeed, this clinical study realized in acute myocardial injury showed a reduction in infarct size with lower level of serum creatine kinase, troponin as well as infarct size assessed by MRI, in patients treated with colchicine 5 days [19]. Similarly, our recent clinical trial [21], showed a significant reduction in left ventricular remodeling in the group of patients treated with colchicine. This cardioprotective effect on infarct size was accompanied by a systemic anti-inflammatory effect characterized by a decrease in cytokines implicated in post-ischemic inflammatory response such as IL 6 and MCP-1. Inflammation is known to exert deleterious effects during acute phase of myocardial infarction with an increase in pro-thrombotic phenomenon [25], in endothelial dysfunction [26] and is associated with adverse outcomes [27,28]. Some inflammatory cytokines, as MCP-1, play an important role in inflammatory cells recruitment $[29,30]$. Indeed, MCP-1 level is known to increase in several experimental myocardial infarction models [31] and to play a major role in leukocyte recruitment, angiogenesis, inflammation activation and resolution. In a rat model of MI, an anti-MCP-1 therapy decreased infarct size presumably through a decrease in adhesion molecule expression and myocardial infiltration by macrophages [32]. Similarly, in a gene therapy study targeting MCP-1, the authors showed an attenuation of left ventricular dilatation in a murine model of myocardial infarction, suggesting an important role of this cytokine in the post-infarction cardiac remodeling [33].

Other interleukins increase after myocardial infarction. IL-10 plays a major role in post-infarction inflammation resolution by inhibiting IL- 6 secretion, a pro-inflammatory cytokine involved in myocardial ischemia $[34,35]$. Here we demonstrated that colchicine significantly decreases serum levels of the main cytokines implicated in post-infarction inflammation (IL-10, IL-6 et MCP-1) $24 \mathrm{~h}$ after reperfusion, suggesting a positive effect on inflammatory cells recruitment in the acute phase of MI. However, several pro-inflammatory cytokines frequently involved in the acute phase of myocardial infarction (TNF- $\alpha$, IL1- $\beta$, TGF- $\beta \ldots$...) were not detected, probably due to the unique dosage realized at $24 \mathrm{~h}$ after the reperfusion [36].

In addition to acute cardioprotective effect, colchicine also exerted a long-term potential positive effects on cardiac hemodynamic with an
A

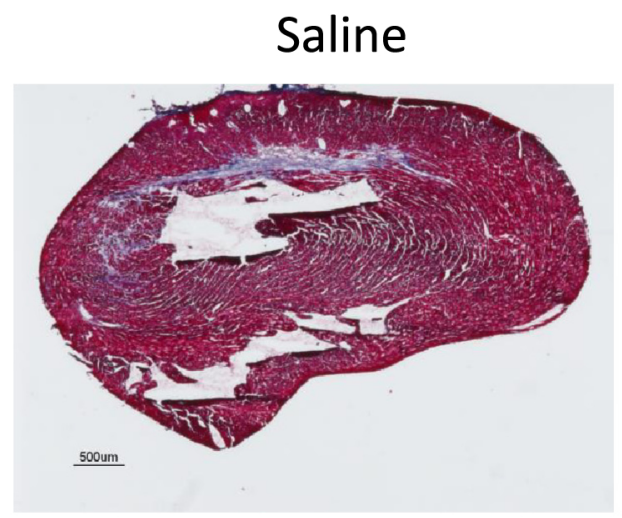

\section{colchicine}

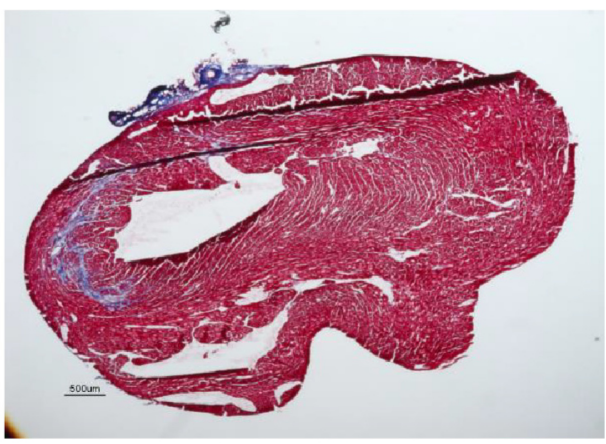

B

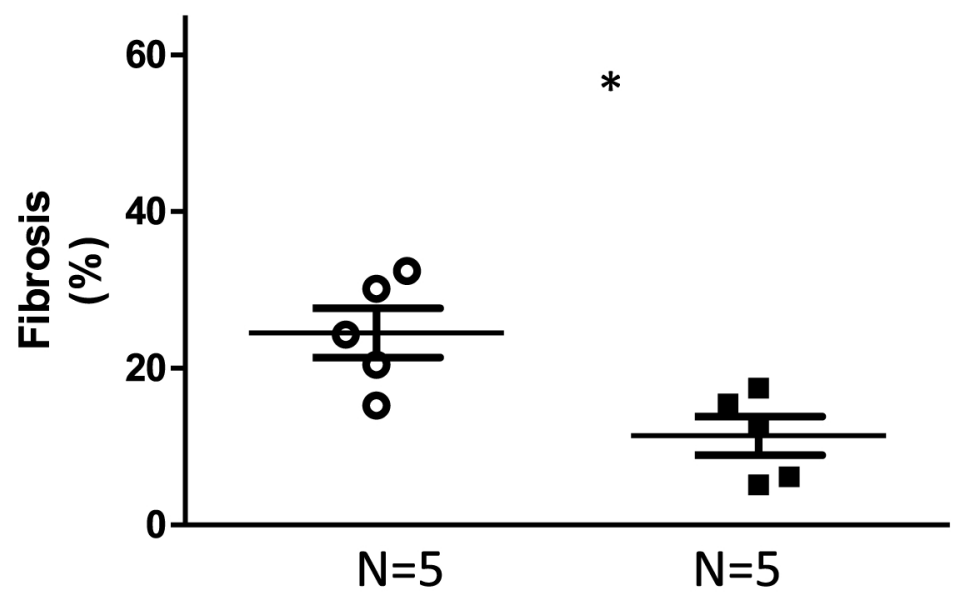

- Saline

- Colchicine

Fig. 4. Fibrosis evaluation with Masson' trichrome coloration A: Heart slices from placebo and colchicine group mice on bifocal microscope after Masson' Trichrome coloration. Fibrosis appears in blue. B: Percentage of fibrosis in heart slices from placebo and colchicine mice. ${ }^{*} \mathrm{p}<0.05$. (For interpretation of the references to colour in this figure legend, the reader is referred to the web version of this article.) 
increase in aortic ITV reflecting cardiac output, on echocardiography realized 8 weeks after AMI. However, usual parameters evaluating the left ventricular diameters (LVEF) were not modified by the treatment with colchicine in the mouse model but were improved in the clinical study. Similar data were found in a clinical study with a reduction in left ventricular remodeling and an improvement in LVEF [19].

Moreover, the histological assessment of fibrosis demonstrates a decrease in post-ischemic myocardial development of fibrosis. Accordingly, this anti-fibrotic effect of colchicine was also demonstrated in a rabbit model of heart failure, reducing fibrosis in left atrium, exerting thus an anti-arrhythmic effect by decreasing the new onset of atrial fibrillation [37]. Although the exact mechanisms remain unclear and might be linked to its anti-inflammatory effect, the anti-fibrotic effect is also largely proven in pericarditis [38] models as well as in several organs, particularly in the liver [39].

\subsection{Study limitation}

The relatively small number of mice dedicated to each exploration is one of the main limitation. However, the adjudication, surgery and analyses were blinded and the main result on infarct size was obtained in 13 animals by group.

Yet, this study aimed to have a first approach on the effect of the colchicine in the acute phase of acute myocardial infarction and at long term, on the evolution toward chronic heart failure. The second limit is the absence of multimodality imaging as cardiac MRI to evaluate differently parameters as infarct size, ventricle remodeling and fibrosis quantification.

The third limitation is the determination of the dose of colchicines in this model. Indeed, preliminary experiments were performed to determine the dose of colchicine used in the animal model (data not shown). A higher dose could indeed exert deleterious effects (narrow therapeutic window in clinical use) but lower dose could be not efficient. A longer or one-shot administration could of course be valuable options. Importantly, the basic and clinical approach cannot be strictly the same route or doses of administrations, hence discrepancing and limiting extrapolation to each other.

Finally, molecular and cellular mechanisms were not extensively explored in this study.

\section{Conclusion}

In this study, a unique dose of colchicine at the early stage of myocardial infarction decreases myocardial injuries with a reduction in infarct size and $\mathrm{T}$ troponin level at $24 \mathrm{~h}$, similarly to recent clinical data. These cardioprotective effects are probably linked to the limitation of the systemic inflammation via the inhibition of the main inflammatory cytokines implicated in the acute phase of myocardial infarction. More importantly, colchicine improves long term cardiac remodeling with improved hemodynamic parameters and reduced myocardial fibrosis. Thus colchicine appears as a promising new therapeutic strategy for chronic ischemic heart failure.

\section{Conflict of interest}

The authors report no relationships that could be construed as a conflict of interest.

\section{References}

[1] J.J.V. McMurray, S. Adamopoulos, S.D. Anker, A. Auricchio, M. Böhm, K. Dickstein, et al., ESC guidelines for the diagnosis and treatment of acute and chronic heart failure 2012: The Task Force for the Diagnosis and Treatment of Acute and Chronic Heart Failure 2012 of the European Society of Cardiology. Developed in collaboration with the Heart Failure Association (HFA) of the ESC, Eur. J. Heart Fail. 14 (8) (Aug 2012) 803-869.
[2] A.L. Bui, T.B. Horwich, G.C. Fonarow, Epidemiology and risk profile of heart failure, Nat. Rev. Cardiol. 8 (1) (Jan 2011) 30-41.

[3] F. Arslan, D.P. de Kleijn, G. Pasterkamp, Innate immune signaling in cardiac ischemia, Nat. Rev. Cardiol. 8 (5) (May 2011) 292-300.

[4] F. Roubille, A. Lacampagne, New drug avenues for cardioprotection in patients with acute myocardial infarction, Am. J. Cardiovasc. Drugs 14 (1) (Feb 2014) 73-77.

[5] F. Roubille, J.-C. Tardif, Inflammation and the heart - prime time for new therapeutic approaches, Expert Opin. Emerg. Drugs 18 (3) (Sep 2013) 259-261.

[6] A. Bonaventura, F. Montecucco, F. Dallegri, Cellular recruitment in myocardial ischaemia/reperfusion injury, Eur. J. Clin. Investig. 46 (6) (Jun 2016) 590-601.

[7] J.A. Urbano-Moral, J.E. Lopez-Haldon, M. Fernandez, F. Mancha, A. Sanchez, M.J Rodriguez-Puras, et al., Prognostic value of different serum biomarkers for left ventricular remodelling after ST-elevation myocardial infarction treated with primary percutaneous coronary intervention, Heart 98 (15) (Aug 2012) 1153-1159.

[8] F. Roubille, D. Busseuil, N. Merlet, E.A. Kritikou, E. Rhéaume, J.-C. Tardif, Investigational drugs targeting cardiac fibrosis, Expert. Rev. Cardiovasc. Ther. 12 (1) (Jan 2014) 111-125.

[9] F. Roubille, E. Kritikou, D. Busseuil, S. Barrere-Lemaire, J.-C. Tardif, Colchicine: an old wine in a new bottle? Antiinflamm. Antiallergy Agents Med. Chem. 12 (1) (2013) 14-23.

[10] K.H. Downing, Structural basis for the interaction of tubulin with proteins and drugs that affect microtubule dynamics, Annu. Rev. Cell Dev. Biol. 16 (2000) 89-111.

[11] R.B.G. Ravelli, B. Gigant, P.A. Curmi, I. Jourdain, S. Lachkar, A. Sobel, et al., Insight into tubulin regulation from a complex with colchicine and a stathmin-like domain, $\mathrm{Na}$ ture 428 (6979) (Mar 11 2004) 198-202.

[12] B. Bhattacharyya, D. Panda, S. Gupta, M. Banerjee, Anti-mitotic activity of colchicine and the structural basis for its interaction with tubulin, Med. Res. Rev. 28 (1) (Jan 2008) 155-183.

[13] R.M. Pope, J. Tschopp, The role of interleukin-1 and the inflammasome in gout: implications for therapy, Arthritis Rheum. 56 (10) (Oct 2007) 3183-3188.

[14] B.N. Cronstein, Y. Molad, J. Reibman, E. Balakhane, R.I. Levin, G. Weissmann, Colchicine alters the quantitative and qualitative display of selectins on endothelial cells and neutrophils, J. Clin. Invest. 96 (2) (Aug 1995) 994-1002.

[15] T.J. Lampidis, D. Kolonias, N. Savaraj, R.W. Rubin, Cardiostimulatory and antiarrhythmic activity of tubulin-binding agents, Proc. Natl. Acad. Sci. U. S. A. 89 (4) (Feb 15 1992) $1256-1260$

[16] K. Saji, Y. Fukumoto, J. Suzuki, S. Fukui, J. Nawata, H. Shimokawa, Colchicine, a microtubule depolymerizing agent, inhibits myocardial apoptosis in rats, Tohoku J. Exp. Med. 213 (2) (Oct 2007) 139-148.

[17] S.I. Rennard, P.B. Bitterman, T. Ozaki, W.N. Rom, R.G. Crystal, Colchicine suppresses the release of fibroblast growth factors from alveolar macrophages in vitro. The basis of a possible therapeutic approach to the fibrotic disorders, Am. Rev. Respir Dis. 137 (1) (Jan 1988) 181-185.

[18] R. Forrat, L. Sebbag, R. Ferrera, G. Hadour, E. Canet, A. Tabib, et al., Effect of colchicine on circulating and myocardial neutrophils and on infarct size in a canine model of ischemia and reperfusion, J. Cardiovasc. Pharmacol. 27 (6) (Jun 1996) 876-883.

[19] S. Deftereos, G. Giannopoulos, C. Angelidis, N. Alexopoulos, G. Filippatos, N Papoutsidakis, et al., Anti-inflammatory treatment with colchicine in acute myocardial infarction: a pilot study, Circulation 132 (15) (Oct 13 2015) 1395-1403.

[20] M. Akodad J. Fauconnier, P. Sicard, F. Huet, F. Blandel, A. Bourret, Barbara P. de Santa et al., Determination of the doses of colchicine used in a study on the impact of colchicine in the treatment of acute myocardial infarct responsible for heart failure in a mouse model, Data Brief (2017) (in press).

[21] M. Akodad, B. Lattuca, N. Nagot, V. Georgescu, M. Buisson, J.-P. Cristol, et al., COLIN trial: interest of COLchicine in the treatment of patients with acute myocardial INfarction and with inflammatory response, Arch. Cardiovasc. Dis. 1875-2136 (16 (Jan 3 2017) 30212-1.

[22] F. Fernandes, F.J. Ramires, B.M. Ianni, V.M. Salemi, A.M. Oliveira, F.G. Pessoa, et al., Ef fect of colchicine on myocardial injury induced by Trypanosoma cruzi in experimental Chagas disease, J. Card. Fail. 18 (2012) 654-659.

[23] P. Mery, B. Riou, D. Chemla, Y. Lecarpentier, Cardiotoxicity of colchicine in the rat, Intensive Care Med. 20 (1994) 119-123.

[24] R. Tochinai, M. Ando, T. Suzuki, K. Suzuki, Y. Nagata, C. Hata, et al., Histopathologica studies of microtubule disassembling agent-induced myocardial lesions in rats, Exp. Toxicol. Pathol. 65 (2013) 737-743.

[25] M. Sadowski, M. Ząbczyk, A. Undas, Coronary thrombus composition: links with inflammation, platelet and endothelial markers, Atherosclerosis 237 (2) (Dec 2014) 555-561.

[26] C. Adlbrecht, R. Wurm, M. Humenberger, M. Andreas, B. Redwan, K. Distelmaier et al., Peri-interventional endothelin-a receptor blockade improves long-term outcome in patients with ST-elevation acute myocardial infarction, Thromb. Haemost. 112 (1) (Jul 3 2014) 176-182.

[27] S. Van Diepen, L.K. Newby, R.D. Lopes, A. Stebbins, V. Hasselblad, S. James, et al. Prognostic relevance of baseline pro- and anti-inflammatory markers in STEMI: an APEX AMI substudy, Int. J. Cardiol. 168 (3) (Oct 3 2013) 2127-2133.

[28] C. Bouleti, T. Mathivet, J.-M. Serfaty, N. Vignolles, E. Berland, C. Monnot, et al., Angiopoietin-like 4 serum levels on admission for acute myocardial infarction are associated with no-reflow, Int. J. Cardiol. 187 (2015) 511-516.

[29] C. Gerard, B.J. Rollins, Chemokines and disease, Nat. Immunol. 2 (2) (Feb 2001) $108-115$.

[30] A.G. Kumar, C.M. Ballantyne, L.H. Michael, G.L. Kukielka, K.A. Youker, M.L. Lindsey, et al., Induction of monocyte chemoattractant protein-1 in the small veins of the ischemic and reperfused canine myocardium, Circulation 95 (3) (Feb 4 1997) 693-700.

[31] N.G. Frangogiannis, C.W. Smith, M.L. Entman, The inflammatory response in myocardial infarction, Cardiovasc. Res. 53 (1) (Jan 2002) 31-47. 
[32] K. Ono, A. Matsumori, Y. Furukawa, H. Igata, T. Shioi, K. Matsushima, et al., Prevention of myocardial reperfusion injury in rats by an antibody against monocyte chemotactic and activating factor/monocyte chemoattractant protein-1, Lab. Investig. 79 (2) (Feb 1999) 195-203.

[33] S. Hayashidani, H. Tsutsui, T. Shiomi, M. Ikeuchi, H. Matsusaka, N. Suematsu, et al., Anti-monocyte chemoattractant protein-1 gene therapy attenuates left ventricular remodeling and failure after experimental myocardial infarction, Circulation 108 (17) (Oct 28 2003) 2134-2140.

[34] N.G. Frangogiannis, L.H. Mendoza, M.L. Lindsey, C.M. Ballantyne, L.H. Michael, C.W. Smith, et al., IL-10 is induced in the reperfused myocardium and may modulate the reaction to injury, J. Immunol. 165 (5) (Sep 1 2000) 2798-2808.

[35] N.G. Frangogiannis, L.H. Mendoza, M. Lewallen, L.H. Michael, C.W. Smith, M.L. Entman, Induction and suppression of interferon-inducible protein 10 in reperfused myocardial infarcts may regulate angiogenesis, FASEB J. 15 (8) (Jun 2001) $1428-1430$.
[36] J. Fauconnier, A.C. Meli, J. Thireau, S. Roberge, J. Shan, Y. Sassi, et al., Ryanodine receptor leak mediated by caspase- 8 activation leads to left ventricular injury after myocardial ischemia-reperfusion, Proc. Natl. Acad. Sci. U. S. A. 108 (32) (Aug 9 2011) 13258-13263.

[37] R. Singhal, S.-L. Chang, E. Chong, Y.-W. Hsiao, S.-H. Liu, Y.-N. Tsai, et al., Colchicine suppresses atrial fibrillation in failing heart, Int. J. Cardiol. 176 (3) (Oct 20 2014) 651-660.

[38] G.C. Clare, R.W. Troughton, Management of constrictive pericarditis in the 21st century, Curr. Treat. Options Cardiovasc. Med. 9 (6) (Dec 2007) 436-442.

[39] N. Nikolaidis, J. Kountouras, O. Giouleme, V. Tzarou, O. Chatzizisi, K. Patsiaoura, et al, Colchicine treatment of liver fibrosis, Hepato-Gastroenterology 53 (68) (Apr 2006) 281-285. 